\title{
Transient simulation of moving ion clouds in time-of-flight ion mobility spectrometers operating with $D C$ and $A C$ fields
}

\author{
M. Allers ${ }^{1 *}$, A. Bohnhorst ${ }^{1}$, A. T. Kirk ${ }^{1}$, B. Ungethüm² ${ }^{2}$ A.Walte ${ }^{2}$, S. Zimmermann¹ \\ ${ }^{1}$ Leibniz Universität Hannover, Institute of Electrical Engineering and Measurement Technology, \\ Department of Sensors and Measurement Technology, Appelstr. 9A, 30167 Hannover, Germany \\ ${ }^{2}$ Airsense Analytics GmbH, Hagenower Str. 73, 19061 Schwerin, Germany \\ * Corresponding author: allers@geml.uni-hannover.de
}

\begin{abstract}
Ion mobility spectrometers can be divided by their principle of ion separation. For example, classical drift tube time-of-flight Ion Mobility Spectrometers (IMS) separate ions by the absolute value of their low field ion mobility; Field Asymmetric Ion Mobility Spectrometers (FAIMS) separate ions by the field dependence of their ion mobility. However, the low field mobilities and the field dependence of the mobility vary only within a limited range for different ions, leading to a limited peak capacity of stand-alone drift tube IMS and FAIMS. Combining both types leads to orthogonal data and thus enhances the selectivity in comparison with stand-alone devices.

In this work, a new approach of enhancing the separation power of a classical drift tube IMS by integrating a field asymmetric waveform ion separation region in longitudinal direction into the drift tube is discussed. This additional separation region is realized by superimposing the constant drift field of a drift tube IMS with an asymmetric parallel AC field using two additional grids inside the drift tube. Since the ions are exposed alternately to high field and low field strengths on their way through the additional separation region, the resulting drift time is affected. Hence, two ion species having the same low-field mobility, but showing a different field dependence of the mobility have different drift times in the enhanced IMS. In order to analyze the ion movement inside such a modified ion mobility spectrometer, the finite element method (FEM) software Comsol Multiphysics is used. Therefore, an existing drift tube IMS model which perfectly agrees with experimental results and considers field inhomogenieties, diffusion, Coulomb repulsion and ion losses at metallic surfaces, is expanded in order to simulate the ion movement in AC fields. This enhanced model provides visualization of the location and shape of the ion cloud during DC/AC operation. Particular attention is given to the increased broadening of the ion cloud due to field inhomogenieties in the additional AC field. Furthermore, ion losses inside the drift tube caused by the AC field and the additional grids are considered. In this work, simulations are used to theoretically investigate our new separation approach to give a first impression of the possible analytical performance.
\end{abstract}

Keywords: FAIMS, Drift Tube Ion Mobility Spectrometer, Field Dependent Ion Mobility, FEM-Model

\section{Introduction}

Ion mobility spectrometers characterize different substances by ionizing the analytes and separating the analyte ions in electric fields. Since IMS operate under atmospheric pressure conditions the instrumental effort is reduced, in particular no vacuum system is required, and compact hand-held systems are available. Due to chemical gas phase ionization processes low detection limits in the ppb or even ppt range can be obtained. These advantages have led to their widespread use as fast detectors for explosives [1], drugs [2], chemical warfare agents, or other hazardous substances [3]. Many other applications have been explored, for example breath gas analysis [4] or quality control [5].

Ion mobility spectrometers can be divided by their principle of ion separation. For example, classical drift tube IMS separate ions due to their different low field ion mobilities $K$ resulting in different drift velocities $v_{D}$ within a constant electric field $E$. A detailed description of IMS fundamentals can be found in [6] or [7].

The ion drift velocity $v_{D}$ is proportional to the electric field $E$ by an analyte-specific ion mobility $K$, as shown in Eq. (1). 


$$
v_{D}=K \cdot E
$$

A fundamental theory of the ion mobility on a molecular scale is given by Revercomb and Mason [8] leading to Eq. (2).

$$
K=\frac{3 e}{16 N} \cdot \sqrt{\frac{2 \pi}{k_{B} T_{e f f}}} \cdot \sqrt{\frac{1}{m}+\frac{1}{M}} \cdot \frac{1}{\Omega_{D}}
$$

In Eq. (2), $k_{B}$ is Boltzmann's constant, $T_{\text {eff }}$ is the effective ion temperature, e is the elementary charge and $N$ is the gas number density. The ion mobility $K$ is a function of the reduced mass calculated from the ion mass $m$ und the neutral mass $M$ as well as their momentum transfer collision cross section $\Omega_{D}$.

At low field and constant ambient conditions the ion mobility $K$ of a specific analyte is constant. However, increasing the electric field the ion mobility becomes dependent on the electric field. The field dependent ion mobility $K(E)$ can be represented by a function $\alpha(E)$ [9], as given in Eq. (3).

$$
K(E)=K \cdot(1+\alpha(E))
$$

The function $\alpha(E)$ may increase or decrease with increasing electric field strength. The increase in the mobility with higher electric field strengths is due to the decrease in ion size, for example because of declustering effects. The decrease in the ion mobility is caused by the dependence of the mobility on the ion temperature, because the collision frequency increases at higher ion temperatures. The ion temperature is influenced by the electric field. Assuming an ideal gas, the mean free path and thus the average kinetic energy gained between two collisions in a constant electric field is inversely proportional to the pressure. Thus, a suitable parameter to describe the kinetic energy according to the applied electric field is the reduced field $E / N . N$ is the gas number density $\left(\right.$ molecules $\left./ \mathrm{cm}^{3}\right)$. The unit of the reduced field is Td (Townsend, $1 \mathrm{Td}=10^{-17} \mathrm{Vcm}^{2}$ ).

Unlike drift tube IMS, FAIMS, also known as differential mobility spectrometry (DMS), separate ions by the field dependence of their ion mobility. The separation of the ions is due to the difference of the ion mobilities at low electric field $K\left(E_{\text {Low }}\right)$ and high electric field $K\left(E_{H i g h}\right)$ which is analyte-specific. This separation method was originally introduced in [9]. A detailed description of the fundamentals of FAIMS is given in [11] or [16]. In FAIMS, ions are introduced by a carrier gas stream into a gap between two closely-spaced electrodes aligned parallel with the axis of the apparatus and the path of travel of the ions. An asymmetric electric field is applied perpendicularly to the carrier gas stream which causes the ions to be deflected perpendicularly alternately to one or the other electrode. The integrated area under the waveform curve for one full period is zero, such that any deviation of the ion trajectory is due to differences in mobility behavior at the two electric field strengths. Ions will either hit one of the electrodes and are neutralized or will pass through the gap for detection. By altering a DC compensation voltage superimposed on the asymmetric electric field, the apparatus can be tuned to select specific ion species. Thus, in contrast to drift tube IMS continuously measuring the total IMS spectrum, FAIMS devices scan the compensation voltage in order to get the total IMS spectrum.

However, due to the limits imposed by ion diffusion, both drift tube IMS and FAIMS are limited in their separation power, especially when thinking of compact hand-held devices. Combining FAIMS and drift tube IMS will take advantage of complementary data on a given ion species and enhance the selectivity in comparison with standalone FAIMS or drift tube IMS. In [12] two drift tube IMS are coupled to a FAIMS system. However, the drift tube IMS can only analyze the ions passed through the FAIMS at a given time. As described above, in order to record the total IMS spectrum, the compensation voltage of the FAIMS system has be swept through a certain range.

In this work, a different approach of combining drift tube IMS and FAIMS will be investigated. The presented method is used in [13] and claimed in [14] as well as [15]. Here, a classical drift tube IMS is equipped with an 
integrated field asymmetric waveform ion separation region. This separation region is realized by superimposing the constant drift field of a drift tube IMS with an asymmetric parallel AC field using two or more additional grids inside the drift tube, like it is shown in Fig. 1. Both asymmetric AC field and DC drift field are oriented in the same direction along the axis of drift tube.

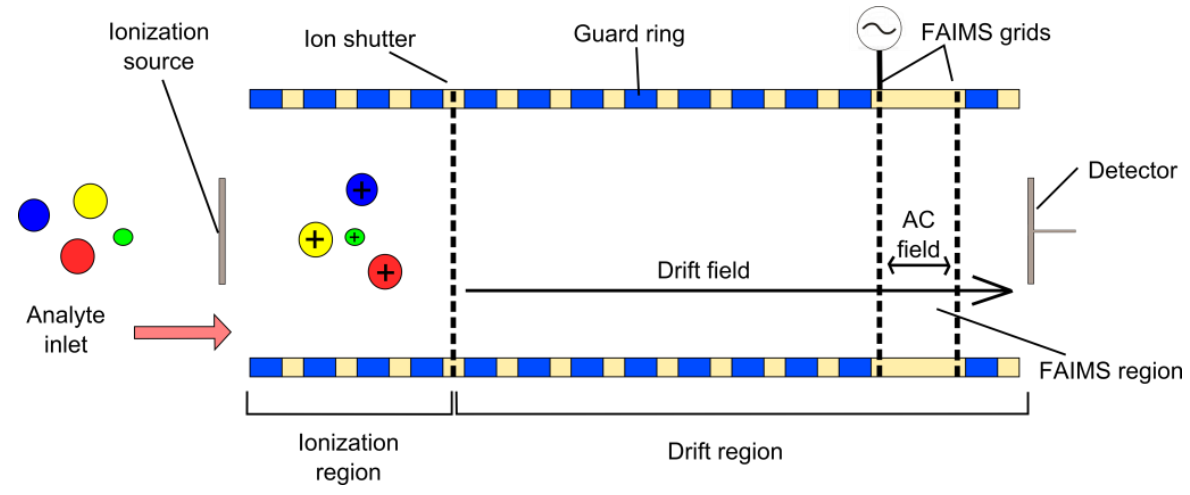

Figure 1: Drift tube IMS with integrated FAIMS region.

The FAIMS enhanced drift tube IMS in Fig. 1 consists of an ionization region, a drift tube and a shielded faraday plate as detector. Ions from the ionization region travel through the drift region under the influence of a low electric drift field generated by metallic drift rings. Now, two additional FAIMS grids are placed inside the drift region and an asymmetric AC voltage is applied to the first FAIMS grid. Due to the constant electric drift field inside the IMS, the ions are drawn through the FAIMS region. Inside this region, the constant electric drift field is superimposed with an asymmetric AC field in longitudinal direction. The integrated area under the waveform curve for one full period is zero and high field strengths alternate with low field strengths. Thus, the constant movement of the ions towards the detector is superimposed with an oscillating movement inside the FAIMS region, as shown in Fig. 2. Ions not showing a field-dependent behavior of the mobility will cover the same additional distance during the high field period and the low field period, but in opposite directions, resulting in no net change of the drift time due to the superimposed oscillating motion. However, ions showing a field-dependent behavior of the mobility will cover different distances during the high field period and the low field period, resulting in a change of the drift time, as they need a different time to traverse the FAIMS region. Depending on the sign of the alpha parameter, the ions cross the FAIMS region faster or slower. Thus, both low-field mobility and fielddependent change of the mobility affect the drift time of the ions in this setup.

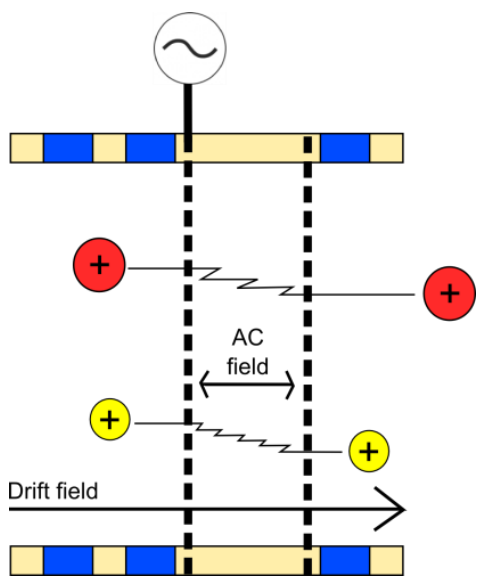

Figure 2: Schematic illustration of the ion movement inside the FAIMS region. In order to visualize the ion movement, a deflection in y-direction is plotted.

In FAIMS or DMS, ion separation occurs due to a combination of AC and DC fields perpendicular to the gas flow drawing the ions through the analytical gap. As a result, ions are separated depending on their alpha parameter only. In the presented approach, ion separation occurs without transport due to gas flow using AC and DC fields which are applied along the axis of a drift tube. As a result, ions are separated depending on their low field mobility 
as well as their alpha parameter. With this new separation method, two ion species having the same low-field mobility, but showing a different field dependence of the mobility have different drift times. Thus, the selectivity can be enhanced in comparison to stand-alone drift tube IMS and FAIMS. Moreover, by switching between the drift tube mode (no additional AC voltage is applied) and the FAIMS mode (an additional AC voltage is applied) orthogonal data on all ion species of the IMS spectrum can be generated.

The additional separation power of the enhanced drift tube IMS is strongly dependent on the properties of the ion species, the size of the FAIMS region and the operating parameters like drift voltage, frequency of the AC voltage, absolute values of $E_{H i g h}$ and $E_{\text {Low }}$ as well as ratio of $E_{\text {High }}$ to $E_{\text {Low }}$ [16]. For example, when neglecting diffusion and increasing the size of the FAIMS region, the separation of two ion species with different $\alpha(E)$ should be increased, since the ions perform more oscillations at a given frequency. However, when increasing the size of the FAIMS region, the applied AC voltages also have to be increased to reach the same electric fields.

In order to obtain a first estimate of the analytical performance of a drift tube IMS with an integrated FAIMS region, we developed a transient simulation which allows a detailed view on the ion movement inside the IMS. For this purpose an existing FEM model of a drift tube IMS, which perfectly agrees with experimental results [17], was expanded in order to simulate the ion movement in AC fields. The model considers all relevant effects, such as field inhomogenieties, diffusion, Coulomb repulsion and ion losses at metallic surfaces. Hence, the simulation can be used to investigate all relevant effects inside a drift tube IMS with an integrated FAIMS region. Particular attention is paid to the increased broadening of the ion cloud due to field inhomogenieties in the AC field. Furthermore, the ion losses inside the drift tube caused by the AC field and the additional grids are considered. In this work, simulations will be used to investigate the separation of two ion species with identical low-field but different high-field mobilities to give first impression of this new separation approach.

\section{Modeling}

The modified IMS is modeled in COMSOL 5.0 using the additional chemical reaction engineering module. This software allows a direct coupling between the calculations of the electrical field and the ion movement. It solves the coupled problem using the finite element method.

The simulated IMS geometry is based on the experimental setup of a drift tube IMS with two additional FAIMS grids as shown in Fig. 1. The IMS consists of an ionization region, a drift tube and a faraday plate as detector. Ionization region and drift region are separated by an ion shutter. The inner diameter of the ionization region and the drift tube is $15 \mathrm{~mm}$. The drift tube has a length of approximately $47 \mathrm{~mm}$ and consists of stainless steel ring electrodes. The distance between ion shutter and first FAIMS grid is $41 \mathrm{~mm}$, the distance between the first FAIMS grid and the second FAIMS grid is $5 \mathrm{~mm}$. The electric drift field $E_{D r i f t}$ between ion shutter and detector is constant. An asymmetric AC voltage is applied to the first FAIMS grid resulting in an asymmetric AC field between the FAIMS grids. The frequency of the AC voltage is in the MHz range. In Table 1 the default values are summarized. Since the focus of this work is on the effects inside the FAIMS region, the ionization region and the ion shutter are not simulated. Instead, a Gaussian ion cloud with an initial width of $10 \mu$ s starts inside the drift tube at $t=0$.

Table 1: Default geometry and operating parameters

\begin{tabular}{|l|l|}
\hline Parameter & Value \\
\hline Length of drift tube & $47 \mathrm{~mm}$ \\
\hline Length of FAIMS region & $5 \mathrm{~mm}$ \\
\hline Inner diameter & $15 \mathrm{~mm}$ \\
\hline Drift field $E_{\text {Drift }}$ & $3 \mathrm{Td}$ \\
\hline Drift voltage & $3,525 \mathrm{~V}$ \\
\hline High field $E_{\text {High }}$ & $75 \mathrm{Td}$ \\
\hline High voltage $U_{\text {High }}$ & $9,375 \mathrm{~V}$ \\
\hline Low field $E_{\text {Low }}$ & $4 \mathrm{Td}$ \\
\hline Low voltage $U_{\text {Low }}$ & $500 \mathrm{~V}$ \\
\hline Frequency of AC field & $0.5 \mathrm{MHz}$ \\
\hline Initial width of ion packet & $10 \mu \mathrm{s}$ \\
\hline
\end{tabular}


As the frequency of the $\mathrm{AC}$ voltage is in the $\mathrm{MHz}$ range, the calculation of the transient ion movement requires very small time differences between two calculation steps. Furthermore, especially near the grids and inside the FAIMS region, the mesh has to be sufficiently fine in order to avoid false results like negative or oscillating ion concentrations. Therefore, a major challenge in the transient modelling of moving ion clouds in AC fields is to reach a reasonable calculation time. In this work, different measures for decreasing the calculation time are applied.

As known from [17], a rotational symmetric geometry as shown in Fig. 3 is used to model the IMS, leading to a significant decrease in calculation time.

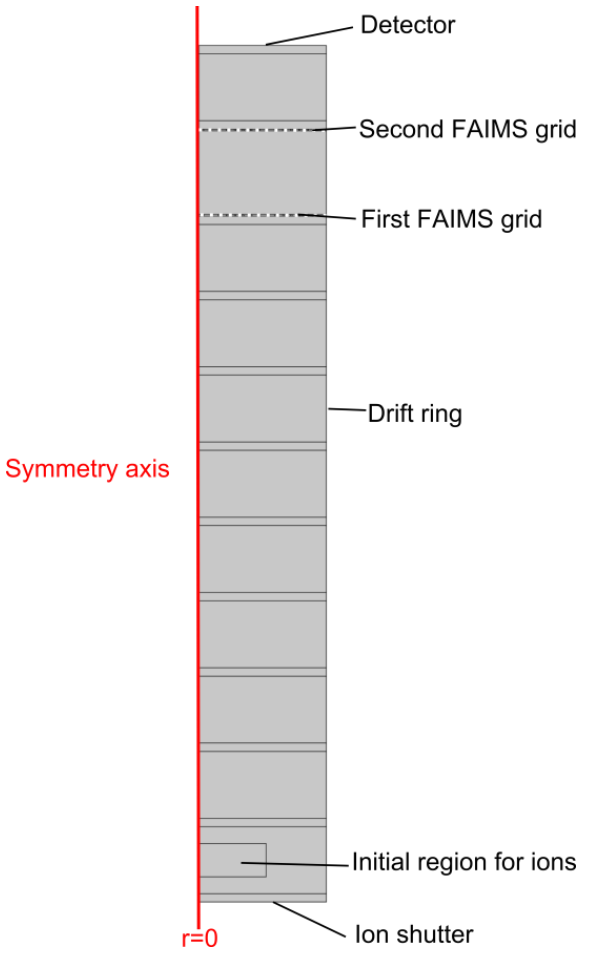

Figure 3: Geometry of the enhanced drift tube IMS in COMSOL 5.0

Furthermore, the division of the domain into finite elements (meshing) is adjusted in order to resolve extreme local electric field gradients or concentration gradients. As already mentioned, this is especially necessary close to the FAIMS grids where high gradients occur. Thus, the entire domain is divided into approximately 300,000 elements. If this mesh would be used, the duration required to calculate the ion movement through the drift tube is more than two weeks. In order to minimize the necessary calculation time, a moving mesh is used. As illustrated in Fig. 4, only the region where the ion cloud is located is accordingly meshed. This way, the used mesh only consists of ca. 60,000 elements, leading to a considerable reduction in the calculation time. 


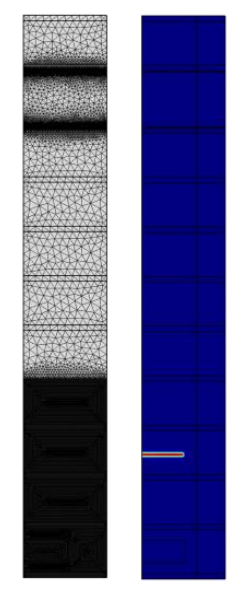

$0<\mathrm{t}<\mathrm{t}_{1}$

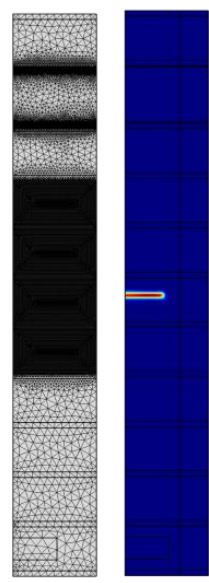

$\mathrm{t}_{2}<\mathrm{t}<\mathrm{t}_{3}$

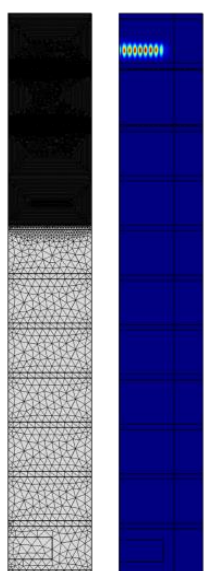

$\mathrm{t}_{4}<\mathrm{t}<\mathrm{t}_{5}$

Figure 4: Moving mesh for the calculation of the ion movement.

The boundary conditions for the calculation of the electric fields are taken from the experimental setup. All electric potentials are constant except for the first FAIMS grid varying from $V_{\text {Low }}$ to $V_{\text {High }}$. In order to further reduce the calculation time, the time dependency of the electric fields is modeled by combining two stationary potential distributions: The potential distribution in the drift tube when no additional AC voltage is applied $\Psi_{\text {Drift }}$ and the potential distribution in the drift tube when the high potential is applied $\Psi_{H i g h}$. Both potential distributions are calculated in the Electrostatics module. The time-dependent potential distribution inside the drift tube $\Psi(t)$ results from a time-dependent step function $S(t)$ periodic in $T$ as given in Eq. (4) and the two stationary potential distributions $\Psi_{\text {Drift }}$ and $\Psi_{\text {High }}$ as shown in Eq. (5).

$$
\begin{gathered}
S(t)=\left\{\begin{array}{cc}
1 & \text { for } 0<t<T \cdot \frac{1}{1+\frac{E_{\text {High }}}{E_{\text {Low }}}} \\
-\frac{E_{\text {Low }}}{E_{\text {High }}} & \text { for } T \cdot \frac{1}{1+\frac{E_{\text {High }}}{E_{\text {Low }}}}<t<T
\end{array}\right. \\
\Psi(t)=\Psi_{\text {Drift }}+S(t) \cdot\left(\Psi_{\text {High }}-\Psi_{\text {Drift }}\right)
\end{gathered}
$$

Thus, the electric field has not to be calculated at every time step by the FEM solver. This also means that the Coulomb repulsion is not considered in this simplified model, which has to be taken into account when discussing simulation results. By applying the measures mentioned above, the calculation time can be decreased from two weeks to two days. However, Coulomb repulsion can be considered if required at the expense of time.

The movement of the ions is calculated in the Transport of Diluted Species module. This module considers migration of the ions in the calculated electric field, diffusion and ion losses at metallic surfaces. In the model, two exemplary ion species with the same low field mobility but different high field behavior are considered. In the model, the ion mobility of the two ion species is defined as a function of the reduced electric field. For ion species 1 a high field $\alpha(E)$ of 0.44 is assumed, for ion species 2 the ion mobility does not change with increasing electric field. The unrealistic $\alpha(E)$ of 0.44 is chosen to better visualize the effect of the AC field on the ion separation.

At high electric fields the diffusion coefficient $D$ is no longer proportional to ion mobility $K$. Thus, the NernstEinstein equation has to be expanded describing also the additional energy supplied from the drift field [10]. In the simulation Eq. (6) is used.

$$
D(E)=\frac{k_{B} T}{e} \cdot K(E) \cdot\left(1+\frac{3 m+M}{5 m+3 M} \cdot \frac{M \cdot v_{D}{ }^{2}}{k_{B} T}\right)
$$


In Eq. (6), $k_{B}$ is Boltzmann's constant, $T$ is the absolute temperature which is $293 \mathrm{~K}, v_{D}$ is the drift velocity, $e$ is the elementary charge, $K(E)$ is the field dependent ion mobility, $m$ is the ion mass and $M$ is the neutral mass.

Any metallic surfaces like the ring electrodes or the grid electrodes are defined as outlets corresponding to ion losses at these boundaries. This ion loss is realized in the simulation by using the boundary condition Flux. The outward flux of an ion concentration $c$ is defined as shown in Eq. (7).

$$
J_{\text {out }}=-K(E) \cdot E \cdot c
$$

\section{Results and discussion}

For the simulations presented in this paper the distance between the FAIMS grids is $5 \mathrm{~mm}$ and the grids have an optical transparency of $85 \%$. The DC drift field has a value of $3 \mathrm{Td}$, corresponding to a drift voltage of $3.525 \mathrm{kV}$. Furthermore, the electric field during the high pulse inside the FAIMS region is set to $75 \mathrm{Td}$, the default value of the low field is $4 \mathrm{Td}$ and a frequency of the $\mathrm{AC}$ voltage of $0.5 \mathrm{MHz}$ is used. With the aid of the simulation, effects on the ion cloud are investigated, when an additional AC field is applied inside the IMS drift tube. This includes the consideration of the field inhomogenieties, the broadening of the ion cloud and possible ion losses at the FAIMS grids. Below, a simulation using the parameter described above is shown. In Fig. 5, the electric field lines inside the FAIMS enhanced drift tube IMS during high and low field conditions as well as the electric field lines in the drift tube mode are compared. For better visualization, the density of the field lines is uniform although the electric field inside the FAIMS region during high field conditions is significantly higher than the original drift field.

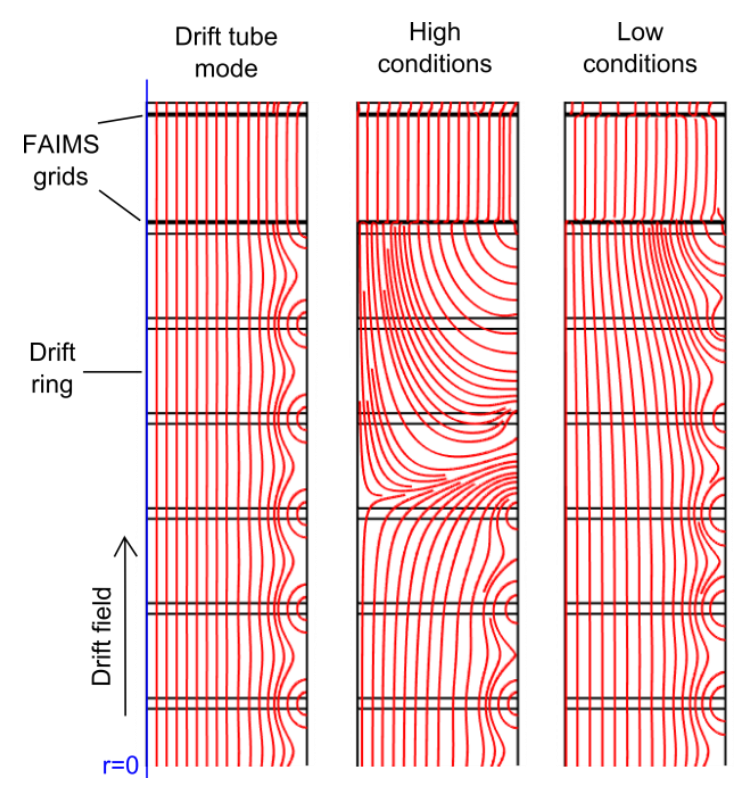

Figure 5: Field distributions inside the IMS: In drift tube mode (left), in FAIMS mode during high field conditions (middle), in FAIMS mode during the low field conditions (right). Drift field: 3 Td, High field: 75 Td, Low field: $4 \mathrm{Td}$.

Especially in the FAIMS mode during the high field conditions, the field lines in the drift tube are strongly deformed. The field lines end on the metallic drift rings in front of the first FAIMS grid meaning that ions cannot enter the FAIMS region. Furthermore, field inhomogenieties in the boundary areas of the tube exist during the low field conditions. Considering the distribution of the potential at the symmetry axis of the tube, the orientation of the electric field becomes visible. During high field conditions a potential barrier prevents positive ions from entering the FAIMS region as shown in Fig. 6. The same happens to positive ions inside the FAIMS region during low field conditions. In this case, a potential barrier prevents them from leaving the FAIMS region. 


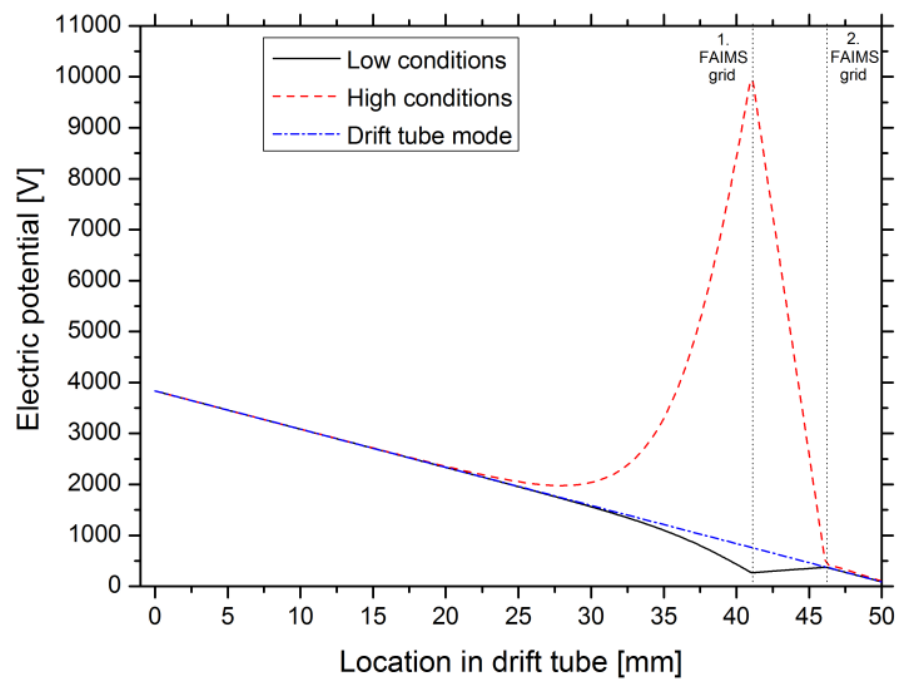

Figure 6: Potential distribution inside the IMS. The strength of the constant drift field is $3 \mathrm{Td}$. Between the two FAIMS grids, the drift field is superimposed with a low field of $4 \mathrm{Td}$ in opposite direction during low conditions and a high field of $75 \mathrm{Td}$ in same direction during high conditions.

The influence of the field inhomogenieties can be illustrated by the simulation of an ion cloud traveling through the FAIMS region. As shown in Fig. 7, the ion cloud is significantly broadened when entering the FAIMS region. Furthermore, the ion cloud is strongly deformed, since ions retard in the boundary areas of the tube due to the electric field.
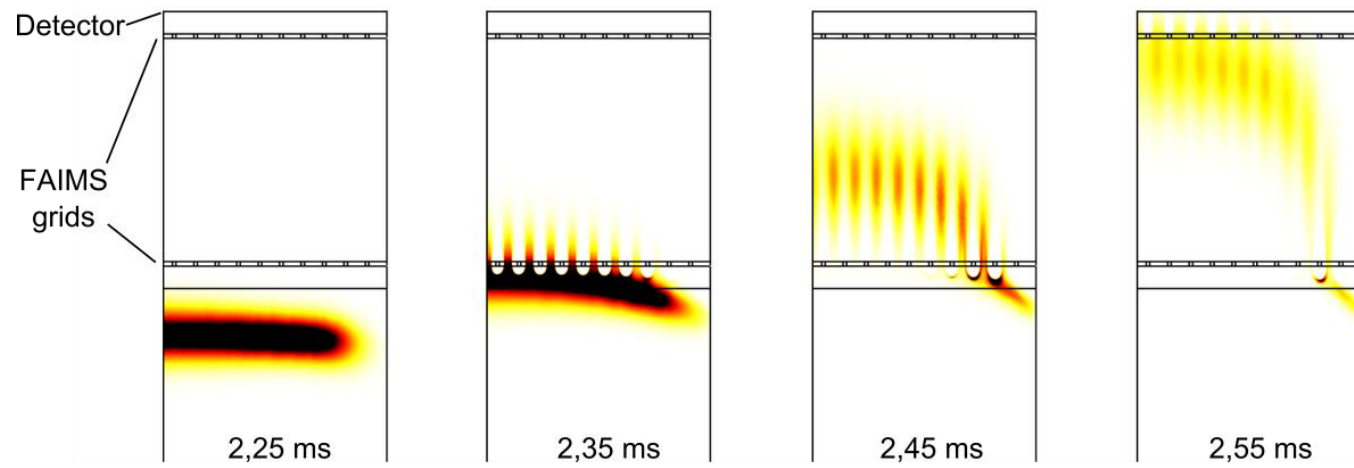

Ion concentration in a.u.

Figure 7: Simulated ion concentration of an ion species traveling through the FAIMS region. Drift field: 3 Td, High field: 75 Td, Low field: 4 Td.

The deformation of the ion cloud leads to a strong tailing of the peak when recorded at the detector, as shown in Fig. 8. 


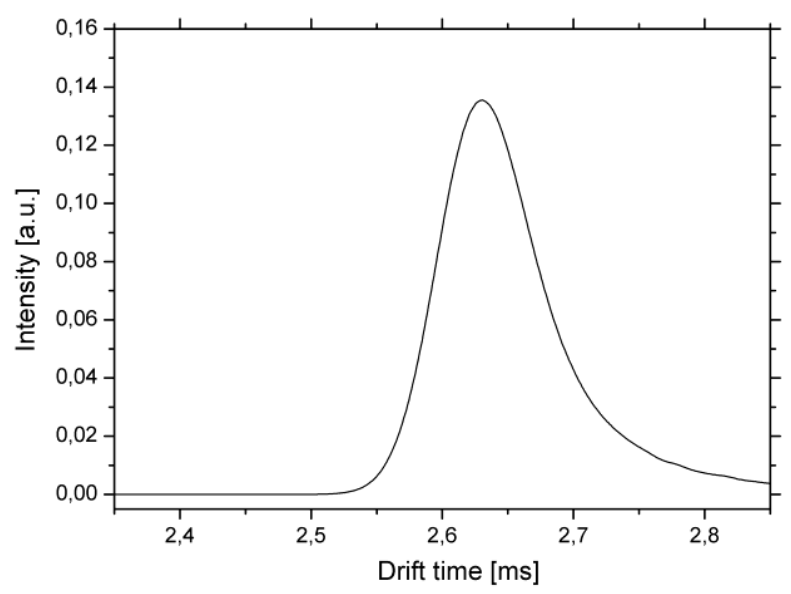

Figure 8: Simulated ion mobility spectrum of an ion species traveling through the FAIMS region. Drift field: 3 Td, High field: 75 Td, Low field: 4 Td.

Moreover, it is evident from Figure 7 that a large number of ions get lost at the FAIMS grids. Fig. 9 demonstrates the ion losses during the movement through the grids. In Fig. 9, the total ion concentration inside the FAIMS enhanced drift tube IMS is plotted against the drift time. It is shown that more than half of the ions get lost at the first FAIMS grid. The ion losses at the second grid are lower.

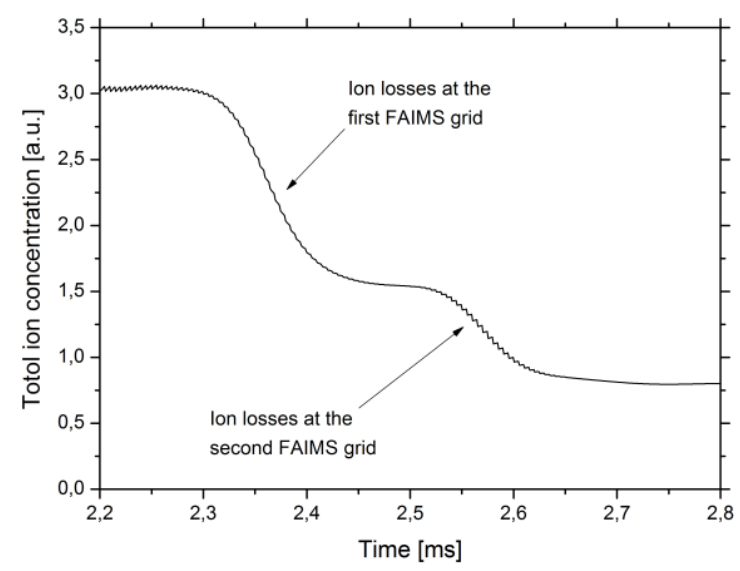

Figure 9: Ion losses during IMS operation: The integrated ion concentration within the IMS during one simulated operation cycle is shown. Drift field: 3 Td, High field: 75 Td, Low field: 4 Td.

In Fig. 10, the simulated IMS spectra of two ion species, assuming the enhanced IMS in the drift tube mode and the FAIMS mode, are compared. As described above, both ion species possess the same low-field mobility, but show a different field dependence of the mobility. Additionally, the IMS spectrum assuming a classical drift tube IMS without additional FAIMS grids is shown. It can be seen that the applied AC field leads to an improvement of the separation of the ion species. In the classical drift tube mode the ion species cannot be separated due to the identical low field mobility. Applying the AC field, both species are shifted slightly. However, it is not sufficient to separate them completely. 

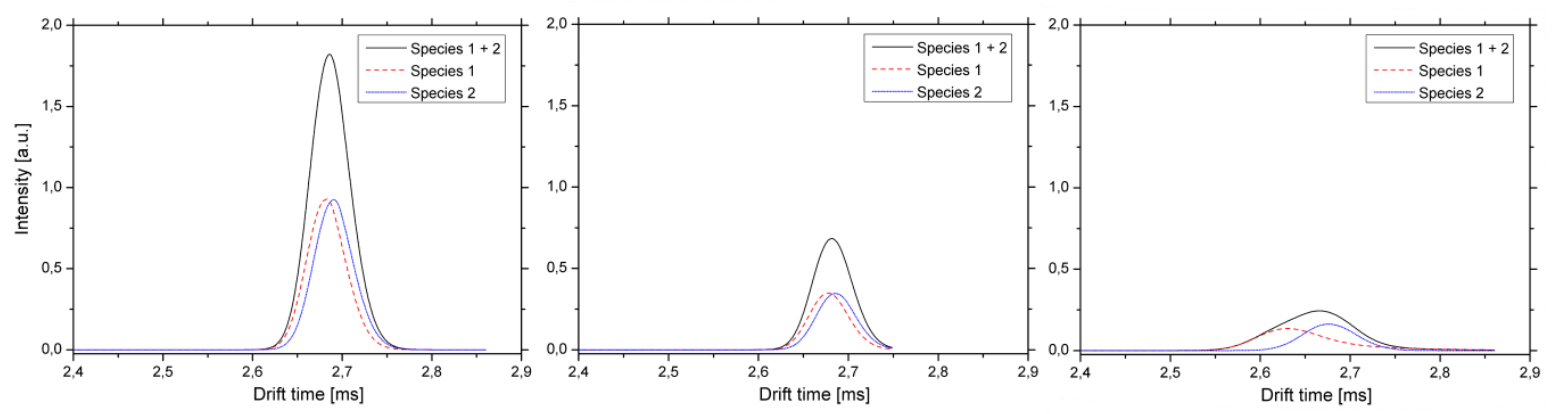

Figure 10: Simulated IMS spectra assuming a classical drift tube IMS (left), a FAIMS enhanced drift tube IMS in drift tube mode (middle) and in FAIMS mode (right). Drift field: 3 Td. In FAIMS mode: High field: 75 Td, Low field: 4 Td.

One reason for the unsatisfactory separation of the two peaks is the strong deformation of the ion cloud when travelling through the FAIMS region, as it is shown above. Furthermore, the ions stay inside the FAIMS region just for a short amount of time. Since this time is not sufficient for the ions to perform a large number of oscillations, the additional separation power due to the field dependent ion mobilities is low. Increasing the size of the FAIMS region would lead to an improvement of the additional separation power, but in practice this would require significantly higher voltages.

Furthermore, considering the simulated IMS spectra in Fig. 10 the decrease in signal intensity becomes obvious. Both the additional two grids in the drift tube and the applied AC field lead to an increase of the ion losses. As shown above, the applied AC field also results in broadening of the ion cloud which also reduces the signal intensity of the peaks. In the simulated example, the signal intensities of the FAIMS enhanced IMS in FAIMS mode are approximately seven times smaller than the signal intensity of a classical drift tube IMS.

Below, the influence of the low reduced electric field strength in the FAIMS region on the separation power and signal intensities is investigated. For this analysis, the high reduced electric field strength remains constant at $75 \mathrm{Td}$ and the low reduced electric field strength is varied. In order to keep the integral electric field values identical, the duty cycle of the waveform must therefore be adjusted. To analyze the separation power, the relative drift time of ion species 1 to ion species 2 is considered. As shown in Fig. 11 left, both an increase and a decrease of the reduced electric low field starting from $4 \mathrm{Td}$ as the default value lead to decreasing separation power. At a reduced low field between $4 \mathrm{Td}$ and $4.5 \mathrm{Td}$ separation power reaches its maximum. In contrast, the signal intensities decrease with increasing reduced low field, see Fig. 11 right, since the ion losses and the broadening of the ion cloud increase. Hence, using this separation approach an improvement of the separation power is always accompanied by a decrease in signal intensity.
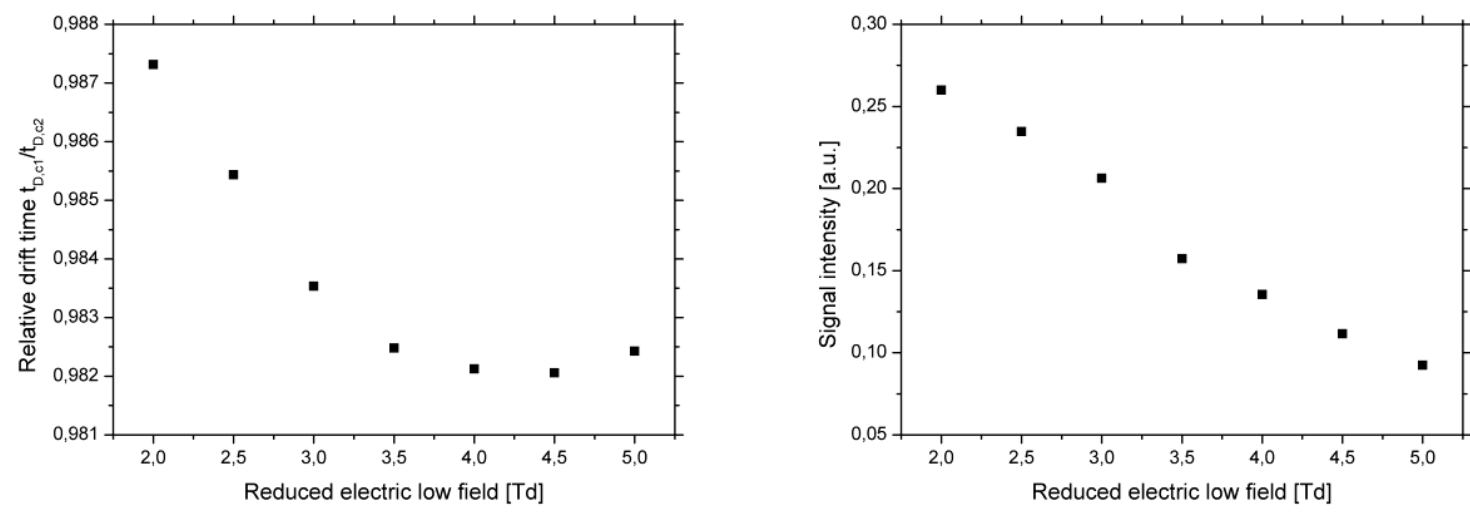

Figure 11: Influence of the reduced electric field in the FAIMS region during low field conditions on the separation power (left) and signal intensities (right). Drift field: $3 \mathrm{Td}$, High field: $75 \mathrm{Td}$. 
As described above, for the simulations presented in this paper the distance between the FAIMS grids is $5 \mathrm{~mm}$ and the reduced electric field during high field conditions inside the FAIMS region is $75 \mathrm{Td}$, corresponding to a voltage of 9,375 V. When evaluating the simulated results, it has to be taken into account that it will be very difficult to design electronic components being able to switch a voltage of approximately $10 \mathrm{kV}$ within microseconds. Furthermore, the two simulated ion species differ widely in their field dependent ion mobility. However, most ion species will differ less, leading to an even lower improvement of the separation power in comparison to the results in Fig. 10. Due to the short residence time of the ions inside the FAIMS region, the observed effects are low. Since an increase of the size of the FAIMS region cannot be implemented in practice because of the required voltages, the effect of the FAIMS region has to be increased in another way. It is conceivable that a setup consisting of more than one FAIMS region results in an increased separation effect. With the help of the simulation, it will be investigated what happens to ions in such a setup. The simulation will be used to estimate, how many FAIMS regions are required to reach a sufficient separation effect. Moreover, the simulation will help to find optimal distances between the FAIMS grids and optimal operation parameters such as voltages or frequency.

Another approach to improve the separation power can be found in the literature. It is well documented [18] [19] that FAIMS separation power in general can be improved significantly by adding chemical modifiers to the transport gas. This is a possibility which can be applied also in the presented setup.

\section{Conclusion}

In this paper, a transient FEM simulation of moving ion clouds in drift tube IMS operating with parallel DC and AC fields is presented. The simulation considers transient electric fields, field dependent ion mobility, ion diffusion and ion losses at electrodes. Hence, the FEM model provides an excellent visualization of the location and shape of the ion cloud during IMS operation. Thus, the simulation is a useful tool to evaluate new approaches in IMS design. In this work, enhancing a classical drift tube IMS by a superimposed field asymmetric waveform ion separation region is analyzed. In the presented method, the constant drift field of the drift tube IMS is superimposed with an asymmetric AC field in longitudinal direction using two additional grids inside the drift tube. This way, complementary data on a given ion species are generated enhancing the selectivity in comparison with stand-alone FAIMS and drift tube IMS devices. With the aid of this simulation, it is shown that the separation of two ion species having identical low-field mobility, but different high field mobilities, can be improved. However, due to field inhomogenieties leading to peak broadening, in the investigated setup, the additional separation power is low. Furthermore, the additional separation power is accompanied by a decrease in signal intensity. Nevertheless, since the simulation results show a positive effect on the separation power, future research concentrates on improved operating parameters and designs of the FAIMS region. For example, it will be investigated if a setup consisting of more than one FAIMS region can increase the reachable separation effect. With the help of the simulation, it will be analyzed what happens to ions in such a setup. Moreover, the simulation will be used to estimate optimal geometry and operation parameters. Furthermore, it is conceivable that the addition of chemical modifiers to the transport gas leads to a significant improvement of the separation power.

\section{Acknowledgement}

This work has been supported by the German Federal Ministry of Economics and Technology (BMWi) under the Grant KF3238301NT3, upon decision of the German Bundestag. 


\section{References}

[1] Ewing, R. (2001): A critical review of ion mobility spectrometry for the detection of explosives and explosive related compounds. In: Talanta 54 (3), S. 515-529. DOI: 10.1016/S0039-9140(00)00565-8.

[2] Hatsis, Panos; Kapron, James T. (2008): A review on the application of high-field asymmetric waveform ion mobility spectrometry (FAIMS) in drug discovery. In: Rapid communications in mass spectrometry: RCM 22 (5), S. 735-738. DOI: 10.1002/rcm.3416.

[3] Eiceman, G. A.; Stone, J. A. (2004): Peer Reviewed: Ion Mobility Spectrometers in National Defense. In: Analytical chemistry 76 (21), S. 390 A. DOI: 10.1021/ac041665c.

[4] Fink, Tobias; Baumbach, Jörg I.; Kreuer, Sascha (2014): Ion mobility spectrometry in breath research. In: Journal of breath research 8 (2), S. 027104. DOI: 10.1088/1752-7155/8/2/027104.

[5] Vautz, W.; Zimmermann, D.; Hartmann, M.; Baumbach, J. I.; Nolte, J.; Jung, J. (2006): Ion mobility spectrometry for food quality and safety. In: Food additives and contaminants 23 (11), S. 1064-1073. DOI: $10.1080 / 02652030600889590$.

[6] Eiceman, Gary Alan; Karpas, Zeev; Hill Jr, Herbert H (2013): Ion mobility spectrometry: CRC Press.

[7] Borsdorf, Helko; Eiceman, Gary A. (2006): Ion Mobility Spectrometry: Principles and Applications. In: Applied Spectroscopy Reviews 41 (4), S. 323-375. DOI: 10.1080/05704920600663469.

[8] Revercomb, H. E.; Mason, E. A. (1975): Theory of plasma chromatography/gaseous electrophoresis. Review. In: Anal. Chem. 47 (7), S. 970-983. DOI: 10.1021/ac60357a043.

[9] Buryakov, I. A.; Krylov, E. V.; Nazarov, E. G.; Rasulev, U.Kh. (1993): A new method of separation of multi-atomic ions by mobility at atmospheric pressure using a high-frequency amplitude-asymmetric strong electric field. In: International Journal of Mass Spectrometry and Ion Processes 128 (3), S. 143148. DOI: 10.1016/0168-1176(93)87062-W.

[10] Mason, Edward A.; McDaniel, Earl W. (1988): Transport Properties of Ions in Gases. Weinheim, FRG: Wiley-VCH Verlag GmbH \& Co. KGaA.

[11] Guevremont, Roger (2004): High-field asymmetric waveform ion mobility spectrometry: A new tool for mass spectrometry. In: Journal of Chromatography A 1058 (1-2), S. 3-19. DOI: 10.1016/j.chroma.2004.08.119.

[12] Anderson, Andrew G.; Markoski, Kenneth A.; Shi, Quan; Coy, Stephen L.; Krylov, Evgeny V.; Nazarov, Erkinjon G. (2008): DMS-IMS2, GC-DMS, DMS-MS: DMS Hybrid Devices Combining Orthogonal Principles of Separation for Challenging Applications. In: Augustus Way Fountain III und Patrick J. Gardner (Hg.): SPIE Defense and Security Symposium. Orlando, FL, Sunday 16 March 2008: SPIE (SPIE Proceedings), S. 69540H.

[13] Miller, R. A.; Kendig, S. D.; Nazarov, E. G.; Coy, S.; Morris, C. J.; Wright, J. A.: Ultra compact ion mobility based analyzer system and method. Applicant: Sionex Corporation 26.07.2005. Application number: PCT/US2006/029283. Patent number: WO 2007/014303 A2.

[14] Atkinson, J. R.; Clark, A.; Grant, B. A. C.; Turner, R. B.: Ion selection apparatus and method. Applicant: Smiths Detection Watford Ltd. 10.01.2006. Application number: PCT/GB2007/000021. Patent number: WO 2007/080376.

[15] Münchmeyer, W.; Ungethüm, B.; Walte, A. (2009): Method and device for detection and identification of gases. Applicant: Airsense Analytics GmbH 12.01.2009. Application number: PCT/DE09/00023. Patent number: WO 2009/089818 A1.

[16] Shvartsburg, Alexandre A. (2009): Differential ion mobility spectrometry. Nonlinear ion transport and fundamentals of FAIMS. Boca Raton: CRC Press. 
[17] Langejuergen, Jens; Cochems, Philipp; Zimmermann, Stefan (2012): Results of a transient simulation of a drift tube ion mobility spectrometer considering charge repulsion, ion loss at metallic surfaces and ion generation 15 (4), S. 247-255. DOI: 10.1007/s12127-012-0095-z.

[18] Schneider, Bradley B.; Covey, Thomas R.; Coy, Stephen L.; Krylov, Evgeny V.; Nazarov, Erkinjon G. (2010): Chemical effects in the separation process of a differential mobility/mass spectrometer system. In: Analytical chemistry 82 (5), S. 1867-1880. DOI: 10.1021/ac902571u

[19] Rorrer, Leonard C.; Yost, Richard A. (2011): Solvent vapor effects on planar high-field asymmetric waveform ion mobility spectrometry. In: International Journal of Mass Spectrometry 300 (2-3), S. 173181 